\title{
Comparison of Grain Boundary Structure in Metals and Semiconductors as Probed by Positrons
}

\author{
J. KURIPLACH \\ Department of Low Temperature Physics, Faculty of Mathematics and Physics, Charles University in Prague \\ V Holešovičkách 2, CZ-180 00 Prague, Czech Republic
}

\begin{abstract}
Vacancy behavior and positron trapping at selected grain boundaries in iron, nickel, and zirconia are investigated theoretically. It is found that the grain boundary vacancy loses its free volume in metals at moderate temperatures whereas it is kept up to very high temperatures in zirconia. The consequences of these findings for positron annihilation studies of nanocrystalline materials are discussed.
\end{abstract}

DOI: $10.12693 /$ APhysPolA.125.722

PACS: 71.60.+z, 61.72.Mm, 61.72.J-, 78.70.Bj

\section{Introduction}

Positron annihilation (PA) spectroscopy (PAS) is very well capable of investigating free volumes in materials. Grain boundaries/interfaces represent an example of regions with reduced atomic density and thereby enlarged interstitial spaces which can be identified with free volumes when compared to perfect bulk material. Grain boundaries (GBs) have been investigated many times using PAS, mainly in nanocrystalline (nc) materials exhibiting a large volume fraction of GBs.

The first idea about what is the origin of trapping sites at GBs in nc materials was formulated in [1] stating that "vacancy-size free volumes" existing at GBs are likely the cause of the lifetime with a magnitude being approximately equal to that of a single vacancy, as observed experimentally for many nc systems. Though such a statement is physically feasible, it has never been proven in detail on the basis of microscopic models of GBs. Experimental PAS studies are valuable to characterize basic defect properties, but the shape of the GB free volumes, where the annihilation of positrons occurs, is not easily accessible. Therefore, it is not clear whether the observed lifetimes are due to true vacancies (i.e. missing atoms in the structure) or some extended free volumes - that are inherent to GBs - the lifetime of which is similar to that of single vacancies.

A coherent ("defect free") GB in a metal possesses some free volume (called often excess free volume) which, however, increases only slightly the positron lifetime [2], and some larger free volume is therefore necessary to explain experimental PA data for nc materials. Previous theoretical studies based on classical molecular dynamics or similar methods (see e.g. $[2,3]$ ) show that vacancies at GBs in metals are not very stable objects and delocalize - i.e. lose their free volume - at rather moderate temperatures of a few hundred $\mathrm{K}$. This means that in real metallic materials all vacancies should be delocalized and no apparent free volumes at GBs should be observed, which is in discrepancy with experimental observations (see e.g. [1, 4]). Nevertheless, this observation agrees with the general idea that GBs serve as a sink for vacancies. On the other hand, perfect GBs in cubic zirconia evidently exhibit free volumes which have a character of channels in the GB plane [5]. Such free volumes can in principle explain experimental positron lifetime data and the presence of vacancies is not needed to form GB free volumes detected in experiment.

In the present work, theoretical studies mentioned will be further extended by performing $a b$ initio molecular dynamics (MD) for selected grain boundaries in metals ( $\mathrm{Ni}$ and $\mathrm{Fe}$ ) and a semiconductor $\left(\mathrm{ZrO}_{2}\right.$ oxide) in order to help understanding the behavior of free volumes at GBs and to clarify the distinction between GB structures in metals and semiconductors (oxides). We chose $a b$ initio MD to confirm some previous results obtained with classical MD and to overcome possible doubts that classical MD is not precise enough to study GBs in materials. Needed positron characteristics will be calculated on the basis of configurations obtained from ab initio calculations.

\section{Computational procedures}

Grain boundaries in materials can be conveniently studied using the so-called coincidence site lattice (CSL) principle $[6,7]$. This principle allows to model GBs in real materials by supposing a special geometrical relationship, i.e. the existence of a CSL, of the two grains between which the considered GB is located. In this way, appropriate periodic supercells can be constructed and handled readily with existing computational first principles codes though the supercell sizes cannot be too large (typically up to a few hundred atoms). Here we study GBs in iron, nickel, and cubic zirconia $\left(\mathrm{ZrO}_{2}\right)$ and compare behavior of vacancies at GBs probed with positrons.

In the case of iron, we have selected a symmetrical tilt $\Sigma 5$ (210) [001] GB for our study. The meaning of the GB designation is as follows: $\Sigma 5$ means that the CSL has 5 times less lattice sites than the original lattice in the same volume, the GB plane is of (210) type and the tilt axis points along an [001] direction in the bcc lattice of Fe. This GB was recently investigated in [8] where five structural modifications of this GB were found. Here, we take the modification with the lowest grain boundary energy (see [8] for details) and show the corresponding supercell in Fig. 1a. 


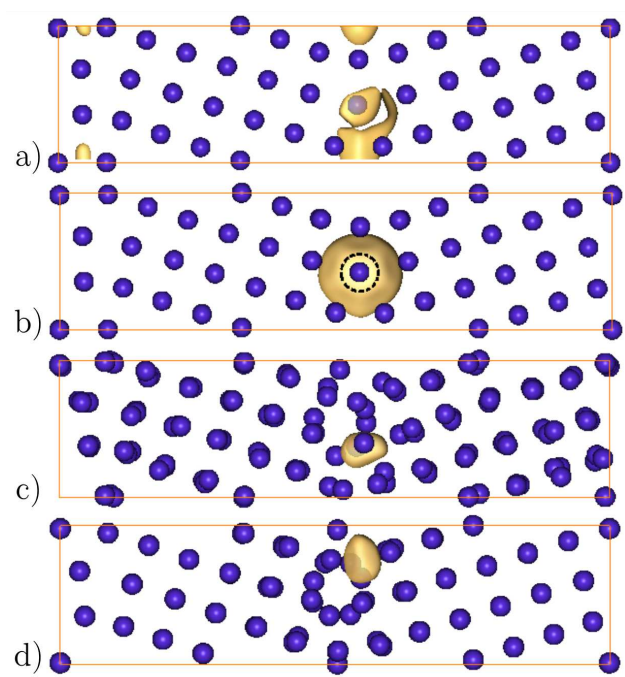

Fig. 1. $\quad \Sigma 5(210)$ GB in Fe. The pictures show the projection of the used supercells along the GB tilt axis: (a) coherent GB configuration, (b) relaxed GB vacancy (vacancy position (projection) is indicated by a dashed circle), (c) one MD snapshot $(800 \mathrm{~K})$ and (d) quenched MD configuration. Light color objects represent the isosurface of the positron density at $50 \%$ of its maximum value.

As for nickel (fcc structure), we considered a symmetrical tilt $\Sigma 19$ (331) [110] GB. The meaning of the GB designation is analogous to that for the Fe GB explained above. This GB has been studied already in [2] using molecular dynamics and the details of $a b$ initio investigations of this GB will be published elsewhere [9]. Here we note that also in this case several GB configurations exist and we choose the one with a mirror plane at the GB. The structure of this GB is visualized in Fig. 2a.

In the case of zirconia (cubic fluorite structure), a symmetrical tilt $\Sigma 5$ (310) [001] GB was taken for investigation. There are already experimental and computational studies of this GB reported in the literature [10, 11]. Two structural modifications of this GB were already examined with positrons in [5] and we study the lower GB energy variant here. The corresponding structure is presented in Fig. 3a.

The CSL constructed supercells contained 120, 228, and 120 atoms for $\mathrm{Fe}, \mathrm{Ni}$, and $\mathrm{ZrO}_{2}$ GBs, respectively. These supercells were optimized with respect to atomic positions using the Vienna $a b$ initio simulation package (VASP) $[12,13]$. The projected augmented wave method [14] was employed. The supercell dimensions perpendicular to GBs studied were also relaxed. In all calculations we employed a generalized gradient approximation for electron exchange-correlations [15].

Vacancies at the studied GB structures were obtained by removing a selected atom from the GB plane (see Figs. 1b, 2b, and 3b). Atomic positions in such configurations were also optimized. Subsequently, ab initio molecular dynamics simulations for GB supercells with vacancies were performed using the VASP code. In particular,

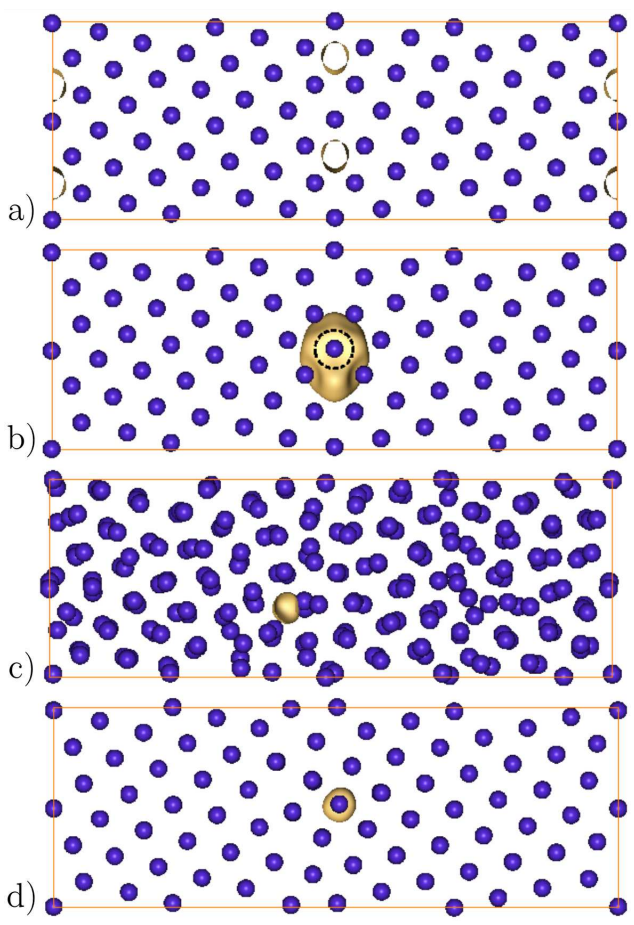

Fig. 2. $\quad \Sigma 19$ (331) GB in Ni. The organization of the figure and color scheme are the same as in Fig. 1: (a) coherent $\mathrm{GB}$, (b) relaxed GB vacancy, (c) one MD snapshot $(2000 \mathrm{~K})$ and $(\mathrm{d})$ quenched MD configuration.

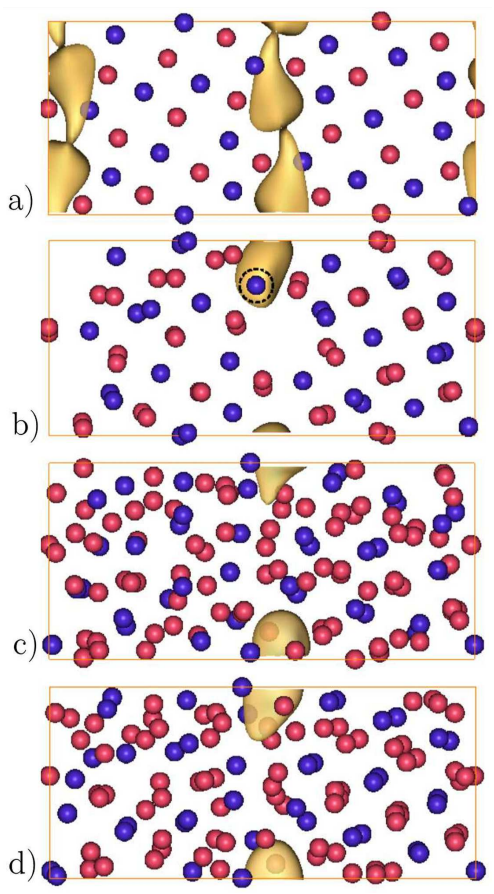

Fig. 3. $\quad \Sigma 5$ (310) GB in $\mathrm{ZrO}_{2}$. The organization of the figure and color scheme are the same as in Fig. 1 except atom coloring ( $\mathrm{Zr}$ : blue (dark) spheres and $\mathrm{O}$ : red (lighter) spheres): (a) coherent GB configuration, (b) relaxed $\mathrm{Zr}$ vacancy configuration, (c) one MD snapshot $(3000 \mathrm{~K})$ and $(\mathrm{d})$ quenched configuration after MD. 
we employed a canonical ensemble with a thermostat after Nosé [16]. The time step was $1 \mathrm{fs}$. The main purpose of these simulations was to expose studied GBs to thermal vibrations and to examine thereby possible vacancy delocalization. Such simulations are quite time consuming and therefore achieving the thermal equilibrium was not the principal goal and will be the subject of future studies. Here, we were mainly interested in detecting the delocalization of vacancies via reduced positron lifetime. This means that the determination of temperature when this effect happens is approximate only and might even exceed the experimentally known melting temperature. After reaching an appropriate temperature - either vacancy delocalization is achieved or the material becomes molten - the supercell is quenched down to zero temperature by stopping thermal atomic movement and relaxing atoms to their equilibrium positions.

Positron calculations were carried out using the atomic superposition method $[17,18]$ as follows. Atomic positions were taken from VASP-produced supercells. According to these positions atomic densities are superimposed and the positron potential is calculated from the electron density. The positron Schrödinger equation is then solved and the ground state positron wave function and energy are obtained. The positron lifetime and binding energy to defects are calculated on the basis of the positron wave function and energy (see review [19] for details). The electron-positron correlation energy and enhancement factor after Drummond et al. [20] were used. These calculations are thus based on the local density approximation and as such they underestimate slightly the calculated lifetimes, typically by a few picoseconds, when compared to experiment. We discuss calculated lifetime values only as there are no experimental data available for specific GBs studied here.

\section{Results and discussions}

\subsection{Grain boundary in iron}

The coherent $\Sigma 5$ (210) GB in Fe gives the positron lifetime $110 \mathrm{ps}$, which is only slightly above the calculated bulk value $99 \mathrm{ps}$. This indicates that the average electron density at the GB is only slightly reduced with respect to the bulk Fe lattice. Despite this observation, the calculated binding energy of positrons to the coherent GB is as large as $1.1 \mathrm{eV}$. One can see in Fig. 1a that the positrons are localized at certain regions of the GB plane. When the vacancy is introduced into the GB plane (Fig. 1b), the positron lifetime increases to $185 \mathrm{ps}$ and the corresponding binding energy amounts to $4.6 \mathrm{eV}$. Though it was not investigated here in detail, a vacancy at a sufficient distance from the GB (i.e. inside one of grains) would have its lifetime and binding energy lower than the corresponding values for a vacancy at the GB [2]. After exposing the defected GB to thermal treatment via $a b$ initio $\mathrm{MD}$, the vacancy delocalizes at about $800 \mathrm{~K}$. One of immediate MD configurations along the evolution trajectory is shown in Fig. 1c. The corresponding positron lifetime is $123 \mathrm{ps}$ indicating an extensive vacancy delocalization. But this shortened lifetime just reflects one immediate configuration. After this configuration is quenched to zero temperature by relaxing atomic positions in the supercell to their equilibrium ones (see Fig. 1d), the resulting lifetime and binding energy become $113 \mathrm{ps}$ and $1.1 \mathrm{eV}$, respectively. These values agree very well with those for the coherent GB, which suggests that the vacancy delocalization is almost complete. We also mention the GB structure change. Figure 1d further shows that there is weak positron localization near the original GB plane and original vacancy position. These effects clearly demonstrate importance of vacancy delocalization phenomena at grain boundaries and that they should be taken into account in PA studies of materials where GBs represent important trapping sites.

\subsection{Grain boundary in nickel}

In the case of the coherent $\Sigma 19$ (331) in Ni (Fig. 2a), the positron lifetime and binding energy were determined to be $124 \mathrm{ps}$ and $1.6 \mathrm{eV}$, respectively. The calculated bulk positron lifetime is $98 \mathrm{ps}$. When comparing these calculated lifetimes with those for the Fe GB (see Sect. 3.1), one can deduce that the density of atoms at the Ni GB is relatively smaller than in the Fe case, which is also supported by the binding energy results. The positron at the GB is localized at channels parallel with the tilt axis (see Fig. 2a). The vacancy introduced into the GB plane exhibits again a strong localization (Fig. 2b) and its lifetime and binding energy were found to be $168 \mathrm{ps}$ and $3.3 \mathrm{eV}$, respectively. $A b$ initio $\mathrm{MD}$ simulations were also performed and one snapshot at $2000 \mathrm{~K}$ is shown in Fig. 2c. This is quite high temperature and very likely the system was not in thermal equilibrium, because it should melt at such temperature, which was not the case. We could not observe vacancy delocalization up to $1500 \mathrm{~K}$ and therefore we had to increase the temperature to $2000 \mathrm{~K}$. Interestingly, vacancy delocalization was observed in [2] at much lower temperatures (below $600 \mathrm{~K}$ ). We also calculated the lifetime and binding energy for the configuration shown in Fig. 2c and obtained $125 \mathrm{ps}$ and $1.8 \mathrm{eV}$, which are values very close to those for the coherent GB. The positron is very weakly localized at a place slightly above the original GB plane (see Fig. 2c). Quenching this configuration to zero temperature results in the structure shown in Fig. 2d. One can observe weak localization at the original vacancy place, and the corresponding lifetime is $145 \mathrm{ps}$. This suggests that a small open volume remains at the GB and, thus, the delocalization of the original vacancy is not complete. The binding energy of positrons to this incompletely delocalized vacancy is $1.9 \mathrm{eV}$ and confirms this conclusion. The GB structure is again changed due to the partly delocalized vacancy.

\subsection{Grain boundary in zirconia}

The coherent $\Sigma 5$ (310) GB exhibits positron localization at the GB region (Fig. 3a), which is also reflected by a positron lifetime of $198 \mathrm{ps}$ (calculated bulk positron lifetime is $135 \mathrm{ps}$ ) and a positron binding energy of $2.7 \mathrm{eV}$. These findings are in line with a more open structure of cubic zirconia (fluorite). If we consider the presence of a Zr vacancy (Fig. 3b), we can see quite large relaxations 
around it. We note that such relaxations were barely visible in the case of $\mathrm{Fe}$ and $\mathrm{Ni}$ GBs with vacancy. The positron lifetime and binding energy are now $235 \mathrm{ps}$ and $3.8 \mathrm{eV}$, which points to a rather deep positron trap like for $\mathrm{Fe}$ and Ni cases discussed above. This means that this vacancy is again well localized. During ab initio MD simulations we could not observe any significant indication of vacancy delocalization till $2500 \mathrm{~K}$. After increasing the temperature to $3000 \mathrm{~K}$, we could observe vacancy delocalization (see Fig. 3c). At the same time, we cannot exclude that the material at the $\mathrm{GB}$ region started melting. The positron is still weakly localized at the original GB area and its lifetime is $192 \mathrm{ps}$ (and binding energy $2.8 \mathrm{eV}$ ). When the supercell is quenched down to zero temperature (Fig. 3d), the structure becomes more ordered, but it does not return to a well ordered state. This effect can be caused by partial melting, by too fast cooling (zirconia can be also made amorphous) or by a structure change since zirconia may exist in several phases. In any case, calculations provide the positron lifetime 221 ps and corresponding binding energy $3.2 \mathrm{eV}$, which corresponds to a slight vacancy delocalization only. This indicates that the $\mathrm{Zr}$ vacancy at the studied GB in zirconia is a very stable defect which can hardly be annealed out.

\section{Conclusions}

Vacancy delocalization was studied in selected symmetrical tilt grain boundaries in nickel, iron, and zirconia by means of $a b$ initio molecular dynamics. We found that the vacancy diminishes its free volume at about $800 \mathrm{~K}$ in the $\Sigma 5$ (210) GB in Fe and at about $2000 \mathrm{~K}$ in the $\Sigma 19$ (331) GB in Ni. On the contrary, the vacancy at the $\Sigma 5$ (310) GB in zirconia remains stable up to high temperatures and the material probably melts at about $3000 \mathrm{~K}$. These findings indicate that in metals possible vacancies should delocalize at rather low temperatures - typically at several hundred $\mathrm{K}$ - whereas in semiconductors (oxides) vacancy free volume may survive till much high temperatures or even to the melting point. A more precise determination of temperatures when vacancies delocalize at GBs using our method is difficult since it is in fact a thermally activated process connected with overcoming some energy barrier in atomic movements. Molecular dynamics is not suitable to obtain precise temperatures when such processes are activated though some workarounds are available (see e.g. [21]). Clearly, further work is needed to understand better the nature of free volumes at GBs in real materials - by considering more materials, GB types, and vacancy positions. In the case of nanocrystalline materials, we still keep our original idea that in metals vacancies at GBs should be delocalized, whereas in semiconductors (at least oxides) they can be stable up to high temperatures. One possibility how to "stabilize" vacancies at GBs in metals could be impurities. But the recent theoretical study of a $\Sigma 5$ (210) GB in Ni [22] indicates that this hypothesis might not be true, at least for this specific GB and examined impurities S and Sb.

\section{Acknowledgments}

This research used resources of the National Supercomputing Center IT4Innovations (Czech Republic), which is supported by the Op VaVpI project number CZ.1.05/ 1.1.00/02.0070. This work was partially supported by the Czech Science Foundation (project P108/12/G043).

\section{References}

[1] H.-E. Schaefer, R. Würschum, Phys. Lett. A 119 , 370 (1987).

[2] J. Kuriplach, O. Melikhova, M. Hou, S.V. Petegem, E. Zhurkin, M. Šob, Appl. Surf. Sci. 255, 128 (2008).

[3] M.R. Sørensen, Y. Mishin, A.F. Voter, Phys. Rev. B 62, 3658 (2000).

[4] J. Čížek, O. Melikhova, I. Procházka, J. Kuriplach, R. Kužel, G. Brauer, W. Anwand, T.E. Konstantinova, I.A. Danilenko, Phys. Rev. B 81, 024116 (2010).

[5] J. Kuriplach, O. Melikhova, J. Č́žzek, I. Procházka, G. Brauer, W. Anwand, Mater. Sci. Forum 733 , 240 (2013).

[6] M.L. Kronberg, F.H. Wilson, Trans. AIME 185, 501 (1949).

[7] P. Lejček, Grain Boundary Segregation in Metals, Vol. 136 of Springer Series in Materials Science, Springer, Heidelberg 2010.

[8] T. Ossowski, J. Kuriplach, E.E. Zhurkin, M. Hou, A. Kiejna, submitted to Phys. Rev. B, 2013.

[9] J. Kuriplach, O. Melikhova, M. Šob, P. Lejček, V. Paidar, to be published, 2014.

[10] E.C. Dickey, X. Fan, S.J. Pennycook, J. Am. Ceram. Soc. 84, 1361 (2001).

[11] S.B. Sinnott, S.Z. Mao, E.C. Dickey, J. Am. Ceram. Soc. 85, 1594 (2002).

[12] G. Kresse, J. Hafner, Phys. Rev. B 47, 558 (1993).

[13] G. Kresse, J. Hafner, Phys. Rev. B 49, 14251 (1994).

[14] P.E. Blöchl, Phys. Rev. B 50, 17953 (1994).

[15] J.P. Perdew, J.A. Chevary, S.H. Vosko, K.A. Jackson, M.R. Pederson, D.J. Singh, C. Fiolhais, Phys. Rev. B 46, 6671 (1992).

[16] S. Nosé, J. Chem. Phys. 81, 511 (1984).

[17] M.J. Puska, R.M. Nieminen, J. Phys. F, Met. Phys. 13, 333 (1983).

[18] A.P. Seitsonen, M.J. Puska, R.M. Nieminen, Phys. Rev. B 51, 14057 (1995).

[19] M.J. Puska, R.M. Nieminen, Rev. Mod. Phys. 66, 841 (1994).

[20] N.D. Drummond, P. Lopéz Ríos, R.J. Needs, C.J. Pickard, Phys. Rev. Lett. 107, 207402 (2011).

[21] A. Ishii, S. Ogata, H. Kimizuka, J. Li, Phys. Rev. B 85, 064303 (2012).

[22] J. Kuriplach, O. Melikhova, M. Šob, submitted to Phys. Rev. B, 2013. 